\title{
Thermobalancing Therapy® should be the First-Line Treatment for Kidney Stones and Benign Prostatic Hyperplasia, Discussions at the Medical Conferences
}

\author{
Simon Allen, MD, PhD, Academician ${ }^{1, *}$ \\ ${ }^{I}$ Director Fine Treatment, Oxford, United Kingdom
}

*Corresponding Author: Simon Allen, MD, PhD, Academician, Director Fine Treatment, Oxford, United Kingdom. Email - info@finetreatment.com

\begin{abstract}
Background: The prevalence of kidney stone disease is increasing around the world, notonly in men but in women. All surgical removals of kidney stones, such as extracorporeal shock wave lithotripsy (ESWL) and percutaneous nephrolithotomy (PCNL) have serious side effects and complication. There are parallel undesirable problems in men after conventional treatments of benign prostatic hyperplasia (BPH) with medications and surgery. At the same time Thermobalancing therapy (TT) enabled by Dr Allen's therapeutic device (DATD) has demonstrated positive impact on chronic internal diseases. Therefore, these issues were discussed at the Nephrology Conference in Spain and at the Global Health Conference in China, in OctoberNovember 2017.The purpose of this review is to talk about the key points of discussions at these conferences and proposals for the future.
\end{abstract}

Methods and Results: The case study on TT with DATD in patient with kidney stones; and the outcomes from the clinical trial on TT in 124 men with BPH, before and after 6-month period they used DATD. TT with DATD dissolved kidney stones in a woman; reduced Prostate Volume $(P V \mathrm{~mL})(P<0.001)$ and diminished urinary symptoms $(P<0.001)$ in men with $B P H$.

Conclusion: The implementations of TT with DATD for the treatment of kidney stone disease were the key note discussions at the Medical Conferences. For over 7 years TT with DATD has been given for hundreds of sufferers with kidney stone disease and thousands with BPH. The results of the case study and clinical trial recommend TT with DATD for kidney stone disease and BPH as the first line treatment. This will help patients to avoid BPH medication and surgical procedures, including ESWL and PCNL, with their risks and complications. Furthermore, DATD should be introduced into the Guidelines for Medical Specialist for men with BPH and people with kidneys stones disease

Keywords: benign prostatic hyperplasia; kidney stone; lower urinary tract symptoms; kidney stone disease management; cause of enlarged prostate; Thermobalancing therapy; Dr Allen's Device

\section{INTRODUCTION}

\subsection{Kidney Stone Disease}

The prevalence of kidney stones in population around the world is high, for instance, in the USA the kidney stones occur in average $8.8 \%$, with difference among men and women, $10.6 \%$, and $7.1 \%$ accordingly; and with the 5-year recurrence rates from $30 \%$ to $50 \%$. [1]. The overall prevalence of kidney stones in China is $7.54 \%, 10.34 \%$ in males and $6.62 \%$ in females [2]. Kidney stone-related prevalence data from 20 countries indicates that the incidence of nephrolithiasis increases globally [3].

There were memos about different methods used on patients with kidney stones in ancient time in Egypt, India, Persia and China and most of them were focused to surgical procedures [4]. However firstly, urinary stones disease in details was described by Hippocrates (460-377 BC), who warned people of using a surgery for removing the stones as wounds of the bladder were lethal [5]. In the 19-century and particularly in 20-century, the first-line treatment of kidney stones has become surgeries [6].

Currently, there is a competition for priority in the treatment of kidney stones between extracorporeal shock wave lithotripsy (ESWL), percutaneous nephrolithotomy (PCNL) and retrograde intrarenal surgery (RIR) [7]. After 
ESWL was implemented it was supposed that $85 \%$ of all patients with kidneys stones could be cured by nonsurgical means [8]. The initial promising results of ESWL were challenge with common side effects caused by this procedure, such as bleeding, severe pain, or urinary tract infections [9], rare complications, such as spleen rupture or intrarenal hematoma $[10,11,12]$, and long-term medical effects: hypertension and diabetes mellitus [13, 14, 15].

PCNL, which has been established in the 1970s to replace open surgery [16], is usually used for removing kidney stones over $2 \mathrm{~cm}$ in size, and may produce different complications, such as bleeding, extravasation, fever which can be treated conservatively, and pleural drain, super selective renal embolization that can be managed minimally invasively if recognised early [17]. Some authors came to conclusion that RIRS can be alternative to PCNL in selected cases [18]. RIRS can be an effective treatment alternative to PNL $[19,20]$. As RIRS affords a comparable success rate to PNL and causes fewer risks of complications it seems to be a promising alternative to PNL when larger stones are to be treated[21, 22].

\subsection{Benign Prostatic Hyperplasia}

Lower urinary tract symptoms (LUTS) secondary to benign prostatic hyperplasia (BPH) most often encountered in men over the age of 50 [23]. In the last decade, the necessity of medical/surgical treatment of BPH has been challenged. BPH/LUTS should not be viewed as an inevitable disease of older people but as part of the aging process that can be prevented [24].

Phytotherapy doesn't significantly improve symptoms of enlarged prostate against placebo, despite being largely safe for ingestion. However, it can be used in patients with mild $\mathrm{BPH}$ symptoms who are reluctant to take standard pharmaceutical medications provided that men understand their current limitations [25].Therefore, recommendations on the evaluation and treatment of LUTS in older men are in demand.BPH treatment should be holistic, and may include conservative measures, lifestyle interventions and behavioural modifications, as well as medication and surgery [26].

\subsection{The Purpose of the Study}

The obtained data about Thermobalancing therapy (TT) enabled by Dr Allen's therapeutic Device (DATD) has shown that innovative therapy is able to treat chronic diseases, such as benign prostatic hyperplasia $(\mathrm{BPH})$ and kidney stone without adverse side effects [27, 28, and 29]. Discussions at the Medical Conferences were aimed at better understanding the origin of the kidney disease and BPH and their treatment.

\section{Methods AND Results}

Dr Simon Allen gave speeches at the $16^{\text {th }}$ European Nephrology Conference, Spain and at the BIT's $5^{\text {th }}$ Global Health Conference in China. He explained the essence of TT. The facts are that the therapy is effective as it tackles the cause of kidney stone disease and prostate enlargement (BPH). The outcomes of the case studies in patients with kidney stones and clinical trial on TT with DATD in men with BPH were presented. This medical innovation was praised by the attending clinicians and scientific researchers from China, USA, United Kingdom, Germany, Spain, France, Italy, Egypt, India, Saudi Arabia, Japan, Indonesia, Australia, and other countries.

\subsection{Study Protocol}

Dr Allen's Device was registered with the Medicines and Healthcare Products Regulatory Agency (MHRA) in the UK in 2010, as a class 1 medical device. A class 1 medical device without a measuring function and supplied in non-sterile condition does not require the involvement of a notified body. So, it is permitted to be used by everyone at home. Therefore, Dr Allen has opened Fine Treatment in the United Kingdom that distributes Dr Allen's Device worldwide.

Today we have satisfied customers from over 100 countries. Ethics committee of the Yerevan State Medical University approved the clinical study on TT and DATD. The study on BPH was registered at the World Health Organisation via the German Clinical Trials Register (DRKS).

\subsection{Study Design}

TT with DATD was used in the clinical controlled studies in The Department of Urology at Yerevan State Medical University. 124 men with BPH and 45 men with CP/CPPS received TT during 6-month period and their clinical parameters before and after therapy were examined. This information was compared with the control groups, i.e. data received from 124 men with BPH who were in watchful waiting. Also, I choose a data of a patient with 
kidney stones that will be presented in this study.

\subsection{The Baseline Evaluations}

It included complete physical examination, medical history, DRE, serum biochemistry, and PSA measurements, electrolytes, urine and renal function tests. Evaluations were made at baseline and 6 months after. The International Prostate Symptom Scale (I-PSS) assessed the dynamics of the BPH patients' conditions. Prostate volume (PV $\mathrm{mL}$ ) was measured by ultrasonography. The standard ellipsoid formula length $\times$ width $\times$ height $\times 0.52$ was used to determine prostate volume. Dynamics of the symptoms and the indicators in each group were evaluated in comparison to their data in the beginning and end of the treatment.

\subsection{Statistical Analyses}

The independent-samples t-test and pairedsamples t-test are suitable only for interval and ratio data, so the Wilcoxon signed-rank test was employed. $\mathrm{P}<0.05$ was considered significant. Statistical analyses were carried out using SPSS v22 (IBM, Armonk, NY, USA).

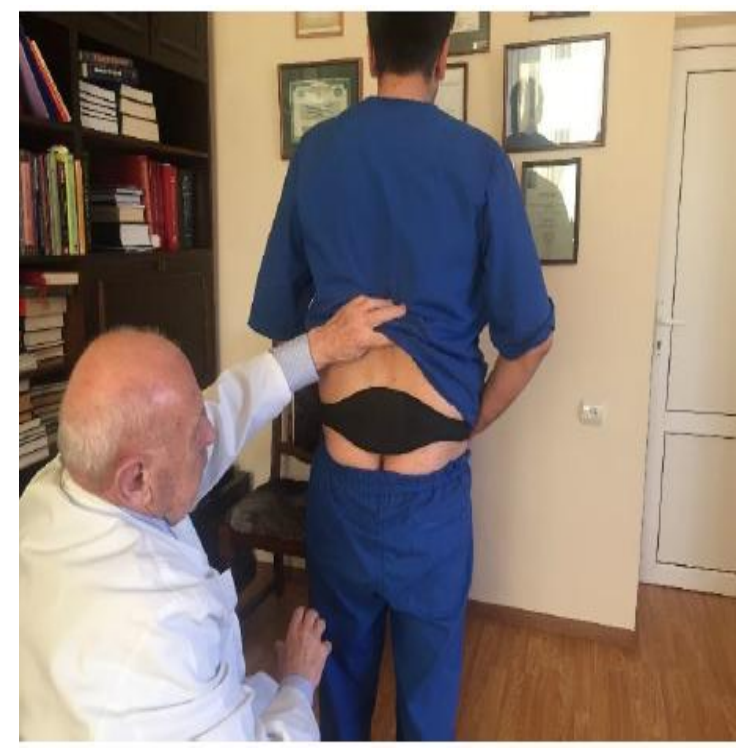

Picture1. DATD tightly attaches thermoelement to the coccyx area for a prolonged period of time.

\subsection{TT and DATD}

TT enabled by DATD for the first time in the world treats the affected organ using own energy of the human body. Natural thermoelement(s) in the device accumulates the body heat and becomes a source of energy itself. Thus, it treats a prostate gland by keeping the source of energy, which has been accumulated from the body, in the projection of the prostate during the prolonged period of time, for 6 months and longer, see Picture

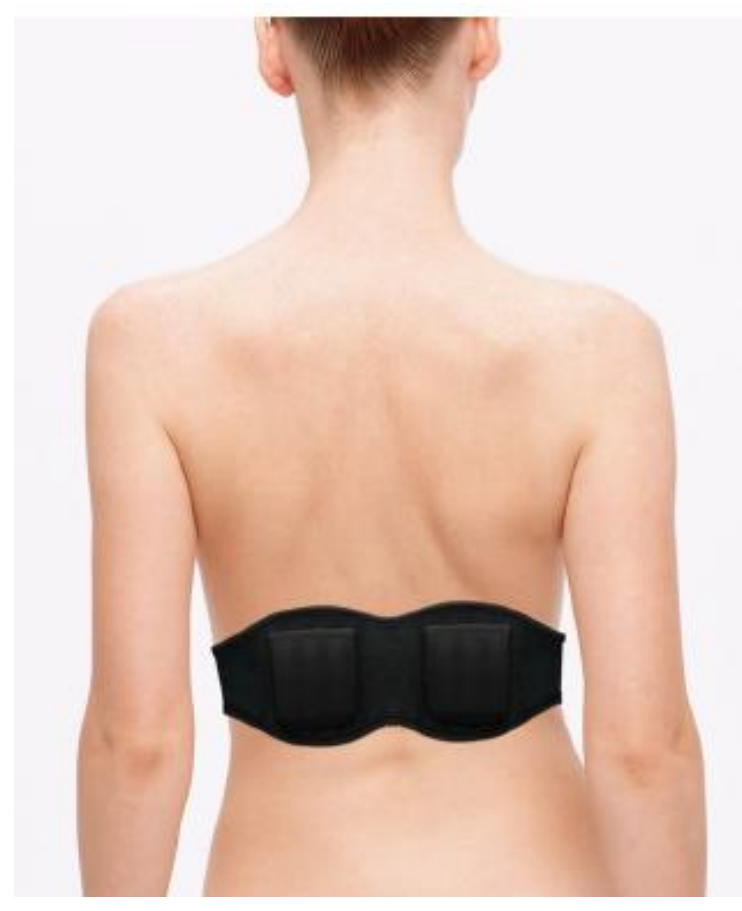

Picture2. Dr Allen's Device for kidney treatment consists of 2 thermoelements and a belt that applies these thermoelements to the projection of both kidneys.

The same happens with patients who suffer with kidney stone disease, when the device applies thermoelements to both kidneys it dissolves stones by keeping the source of energy, which has been accumulated from the body, in the projection of both kidneys during the prolonged period of time, see Picture 2.

\section{RESUlTS}

We have collected a number of case studies provided to me by the users and should note that not a single user of Dr Allen's Device for kidney treatment has complained that this treatment method has not worked for him or her. And this is a very valuable user feedback.

Ruud B, 48, After 2 months of using the device wrote that the $\mathrm{x}$-ray showed a stone of $4 \mathrm{~mm}$ while two months ago they told me it was $8 \mathrm{~mm}$, in 2011.

Frank A, 44, had kidney stones history in one kidney for 20 years. Hestarted use the device in 2012. Has no problem since.

Donald B, 50, kidney stone $8 \mathrm{~mm}$ was found after renal colic recently. He started use the device in 2014. No problems since. 


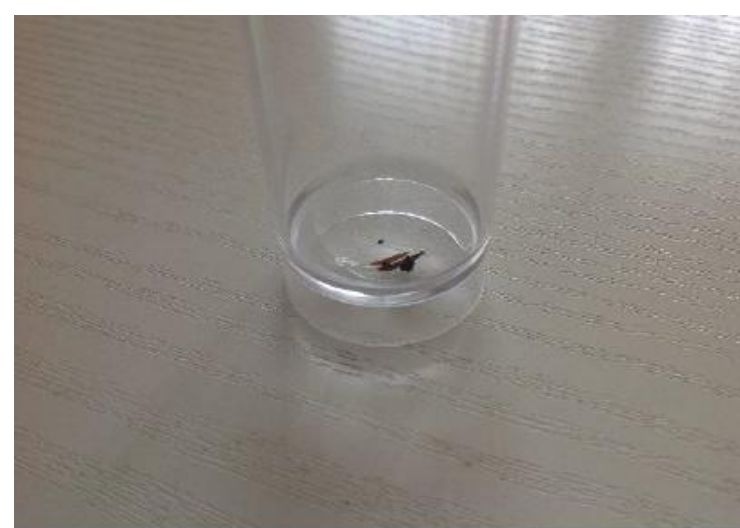

Picture3.The passed kidney stones before Anja started use DADT.Over 2 years ago, she started using Dr Allen's Device. She has not experienced a renal colic since.

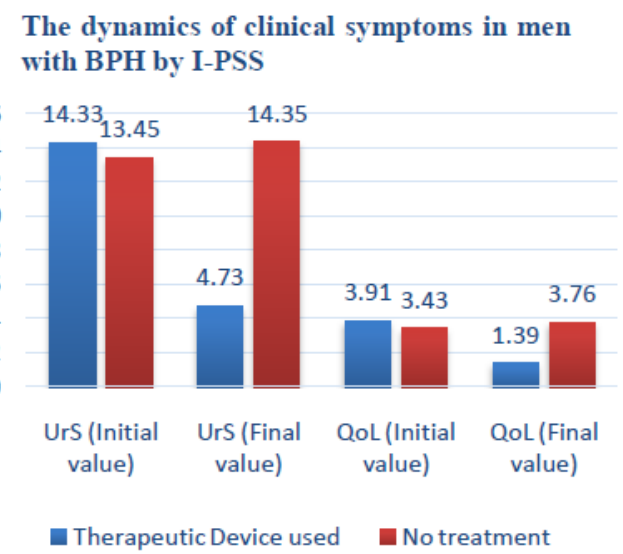

Figure1. Dynamics of the International Prostate Symptom Score (IPSS) for urinary symptoms (UrS) and quality of life $(Q o L)$ in patients with BPH upon Thermobalancing therapy and in the control group at the beginning and at end of the study.

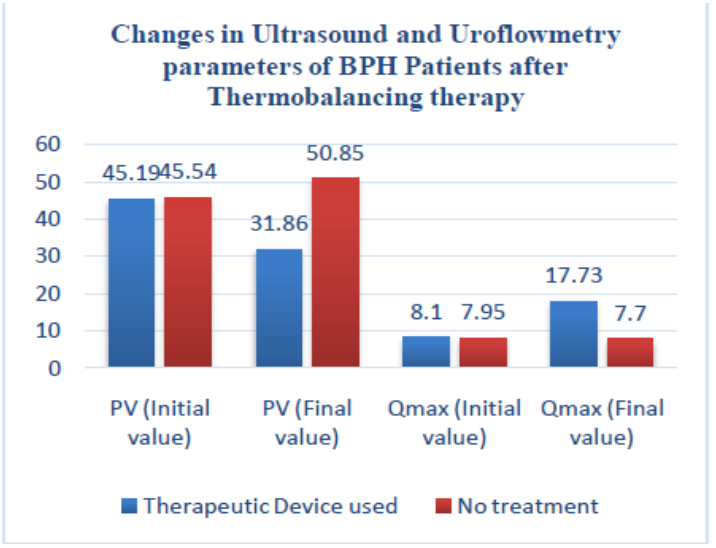

Figure2. Changes in prostate volume $(P V, m L)$ and uroflowmetry (maximum urinary flow rate, $Q_{\max }$ $\mathrm{mL} / \mathrm{s}$ ) in men with BPH upon Thermobalancing therapy and in the control group at the beginning and at end of the study.

Anja A, 44 years of age, had been suffering from kidney stones for 24 years. She used to have renal colic every year until March 2015. She used to collect some of the stones she was passing, and sent a photo of them to me at the start of the treatment, see picture 3 .

The outcomes of the clinical trial on TT in 124 men with BPH are presented in Figure 1 and Figure2.

Clinicians and scientific researchers at the Medical Conferences welcomed new safe treatment option for urological conditions: for kidney stones disease, chronic prostatitis and prostate enlargement.

\section{DISCUSSIONS}

\subsection{The Origin of Diseases, Including Kidney Stones and BPH}

According to a new understanding of the origin of diseases developed by Dr Allen [30], the vascular factor plays a decisive role in the etiology and pathogenesis of all chronic internal diseases. Dr Allen's research reveals that all chronic internal diseases have the same root, namely, a pathological activity of capillaries.

The constriction of capillaries in response to different triggers, such as infection, cold, excess of elements in blood and others creates the focus of hypothermia in the affected tissue, which gradually becomes a trigger itself, irritating the affected tissue. To improve blood circulation in this affected tissue the spontaneous expansion of a capillary net occurs. Slowly the expanded capillaries form extra tissue that creates pressure in this area of the organ leading to its malfunction $[31,32]$.

Thus, the conclusion is that this physical factor is responsible for the development of chronic internal disease. In order to eliminate the focus of hypothermia and, consequently, the pressure inside the organ, and ultimately, its malfunction TT enabled by DATD have been developed.

\subsection{Kidney Stone Disease}

At medical conferences, some cases were discussed that confirmed the effectiveness of TT with DATD in the treatment of kidney stone disease in the last 7 years. There have been patients who have been diagnosed with a kidney stone recently, for example Donald, and those who have suffered from kidney disease for many years.

We see that in the presented case study Anja have suffered with renal colic for 20 years and nothing was able to stop it. Only with the use of 
TT with DATD could improve her QoL as she has no renal colic since. We have many other empirical evidences when even large kidney stones were dissolved. It should be noted that none of patients for seven years has complain that he or she had pain while their stone reduced in size and have gone completely. Moreover, there is preliminary evidence that the therapy can improve kidney function in people with long lasting kidney stone disease.

Nowadays urologists to protect people with kidney stones from side effects and complications after surgical procedures and stones recurrence think out to find a connection between dietary factors and the risk of incidence of this disease [33 34]. Some foods and supplements are associated with higher risk for development of kidney stones [35,36]. To guide patient treatment, it was suggested to define the clinical phenotype of the idiopathic calcium nephrolithiasis, which differs to non-idiopathic calcium stones, uric acid, cystine and struvite stones $[37,38,39]$.

According to the Oxford Centre for Evidencebased Medicine, Jung with college evaluated dietary and medical management of patients with kidney stones by using evidence from the literature suggesting rational dietary and medical management regimes [40].

Themanagement of kidney stones should be evaluated according to severity of side effects and complications after treatments. The dietary and/or medical treatments have never shown possibility to dissolve kidney stones. Therefore, implementation TT with DATD asthe first-line kidney stones treatment into the Guideline for Nephrologists and General Practitioners should be carried out as soon as possible.

\subsection{Benign Prostatic Hyperplasia}

Even though BPH now is considered a consequence of aging, treatment of this disease centered on medical/surgical intervention to counter act LUTS. Medical treatment of BPH is not efficacious as $52.8 \%$ of men with $\mathrm{BPH}$ were dissatisfied with the results of medical treatment conducted according to current international guidelines for BPH [41].

BPH medications have serious side effects, especially in the long-term [42]. Surgical treatments of prostate were found to be unsafe for non-Caucasian race, so for them conservative treatment might be a reasonable alternative [43]. Additionally, the results of a survey of sexually active men after three different laser surgeries from 2005 to 2010, concluded that all these surgical techniques can have a negative impact on sexual function, and patients with normal preoperative sexuality are more at risk [44].

Although many of medical/surgical interventions were advised to men with LUTS, it was generally done in a non-standardised and unsystematic way. A self-management programme could significantly reduce the frequency of escalation through the treatment cascade and reduce urinary symptoms (as effective as medication), suggesting that selfmanagement could be considered as first-line treatment for men with LUTS [45].

The self-management of LUTS due to BPH with the use of DATD demonstrated its high efficacy [46]. It was also proved to be effective in treatment of chronic prostatitis and chronic pelvic pain, which may be responsible for the development of BPH [47, 48]. Therefore, implementation TT with DATD as the first-line BPH treatment into the Guideline for Urologists and General Practitioners should be carried out as soon as possible.

\subsection{TT with DADT Treats the Cause of BPH and the Formation of Kidney Stones.}

Today, with the invention of TT with DATD [49], the quality of life of people with chronic internal diseases such as kidney stones and BPH should be changed positively. The violation of the microcirculation along with emergence of the focus of hypothermia, which itself becomes a continuous trigger, leads to the formation of kidney stones and development of enlarged prostate [50]. Therefore, it is very important to use TT with DATD for the treatment of kidney stones and $\mathrm{BPH}$.

\section{CONCLUSiON}

TT with DATD is an effective tool for the treatment of kidney stone disease and BPH. Kidney stone disease ought to be treated therapeutically by Thermobalancing therapy in the first instance, helping patients to avoid surgical procedures, including lithotripsy, with their risks and complications. The treatment of LUTS due to BPH should be considered carefully and start with DATD, as TT is effective, safe and cost-effective. Therefore, it is important to introduce TT with DATD in the 
Guide for Medical Specialists for the treatment of kidney stones and BPH.

\section{ACKNOWLEDGMENTS}

I am grateful to the organisers and management team of Conference Series and BIT's Conferences in China for giving opportunity to meet medical professionals from different parts of the world.

\section{REFERENCES}

[1] Scales CD Jr, Smith AC, Hanley JM, Saigal CS Prevalence of kidney stones in the United States, EurUrol, 2012, 62(1):160-5. doi: 10.1016/j.eururo.2012.03.052.

[2] Wang W, Fan J, Huang G et al Prevalence of kidney stones in mainland China: A systematic review. Scientific Reports, 2017, 7:41630. doi:10.1038/srep41630.

[3] Romero V, Akpinar H, Assimos DG Kidney Stones: A Global Picture of Prevalence, Incidence, and Associated Risk Factors. Reviews in Urology, 2010; 12(2-3):e86-e96. 4. Shah J, Whitfield HN Urolithiasis through the ages, BJU Int. 2002 May;89(8):801-10.

[4] Shah J, Whitfield HN. Urolithiasis through the ages. BJU International. 2002;89(8):801-810.

[5] Dimopoulos C, Gialas A, Likourinas M, Androutsos G, Kostakopoulos A Hippocrates: founder and pioneer of urology. $\mathrm{Br} \mathrm{J}$ Urol. 1980, 52(2):73-4.

[6] Tefekli A, Cezayirli F The History of Urinary Stones: In Parallel with Civilization, The Scientific World Journal, 2013, 2013:423964. doi:10.1155/2013/423964.

[7] Srisubat A, Potisat S, Lojanapiwat B, Setthawong V, Laopaiboon M Extracorporeal shock wave lithotripsy (ESWL) versus percutaneous nephrolithotomy (PCNL) or retrograde intrarenal surgery (RIRS) for kidney stones. Cochrane Database of Systematic Reviews, 2014, Issue 11. Art. No.: CD007044. DOI: 10.1002/14651858.CD007044.pub3.

[8] Chaussy C, Schmiedt E Extracorporeal shock wave lithotripsy (ESWL) for kidney stones. An alternative to surgery? UrolRadiol, 1984, 6: 80. doi:10.1007/BF02923707.

[9] Skolarikos A, Alivizatos G, De la Rosette J "Extracorporeal shock wave lithotripsy 25 years later: complications and their prevention," European Urology, 2006, 50, 5, 981-990.

[10] White WM, Morris SA, Klein FA, Waters WB Splenic rupture following shock wave lithotripsy. Can J Urol. 2008, 15:4196-4199.

[11] Marinkovic SP, Marinkovic CM, Xie D Spleen injury following left extracorporeal shockwave lithotripsy (ESWL), BMC Urology, 2015, 15:4. doi:10.1186/1471-2490-15-4.

[12] Akbulut F, Kucuktopcu O, Ucpinar B, et al A Rare Complication of Extracorporeal Shock Wave Lithotripsy: Intrarenal Hematoma Mimicking Pelvis RenalisTumor, Case Reports in Urology, 2015, Article ID 719618, 4 pages, doi:10.1155/2015/719618.

[13] KrambeckAE, Gettman MT, Rohlinger AL, Lohse CM, Patterson DE, Segura JW Diabetes Mellitus and Hypertension Associated With Shock Wave Lithotripsy of Renal and Proximal Ureteral Stones at 19 Years of Followup, J Urol, 2006, 175(5):1742-7.

[14] McAteer JA, Evan AP The Acute and LongTerm Adverse Effects of Shock Wave Lithotripsy. Seminars in nephrology, 2008, 28(2):200-213, doi: 10.1016/j.semnephrol.

[15] D'Addessi A, Vittori M, Racioppi M, Pinto F, Sacco E, Bassi PF. Complications of Extracorporeal Shock Wave Lithotripsy for Urinary Stones: To Know and to Manage Them-A Review, The Scientific World Journal, 2012, Article ID 619820, 6. doi:10.1100/2012/619820.

[16] Fernström I, Johansson B Percutaneous pyelolithotomy. A new extraction technique, Scand J UrolNephrol, 1976, 10:257-9.

[17] Michel MS, Trojan L, Rassweiler JJ Complications in percutaneous nephrolithotomy. EurUrol, 2007, 51(4):899906; discussion 906.

[18] Bai Y, Xiaoming Wang X, Yang Y, Han P, Wang J Percutaneous nephrolithotomy versus retrograde intrarenal surgery for the treatment of kidney stones up to $2 \mathrm{~cm}$ in patients with solitary kidney: a single centre experience, BMC Urol, 2017, 17: 9. doi: 10.1186/s12894017-0200-z.

[19] Koyuncu H, Yencilek F, Kalkan M, Bastug Y, Yencilek E, Ozdemir AT Intrarenal Surgery vs Percutaneous Nephrolithotomy in the Management of Lower Pole Stones Greater than $2 \mathrm{~cm}$. International Brazilian Journal of Urology: official journal of the Brazilian Society of Urology, 2015, 41(2):245-251. doi:10.1590/S1677-5538. IBJU.

[20] Sari S, Ozok HU, Cakici MC, Ozdemir H, Bas O, Karakoyunlu N, Sagnak L, Senturk AB, Ersoy H A Comparison of Retrograde Intrarenal Surgery and Percutaneous Nephrolithotomy for Management of Renal Stones $\geq 2$ CM, Urology Journal, 2017, 14, 1, 2949-2954.

[21] HO CCK, Hee TG, Hong GE, Singam P, Bahadzor B, MdZainuddin Z Outcomes and Safety of Retrograde Intra-Renal Surgery for Renal Stones Less Than $2 \mathrm{~cm}$ in Size. Nephro- 
urology monthly, 2012, 4(2):454-457. doi:10.5812/numonthly.2211.

[22] Zengin K, Tanik S, Karakoyunlu N, et al Retrograde Intrarenal Surgery versus Percutaneous Lithotripsy to Treat Renal Stones $2-3 \mathrm{~cm}$ in Diameter, BioMed Research International, 2015, Article ID 914231, 4 pages, doi:10.1155/2015/914231.

[23] Rohrmann S, Katzke V, Kaaks R Prevalence and Progression of Lower Urinary Tract Symptoms in an Aging Population, Urology. 2016 Sep; 95:158-63. doi: 10.1016/ j.urology. 2016.06.021.

[24] Corona G, Vignozzi L, Rastrelli G, Lotti F, Cipriani S, Maggi M. Benign Prostatic Hyperplasia: A New Metabolic Disease of the Aging Male and Its Correlation with Sexual Dysfunctions. Int J Endocrinol. 2014; 2014:329456.

[25] Keehn A, Lowe FC Complementary and alternative medications for benign prostatic hyperplasia, Can J Urol. 2015 Oct;22 Suppl 1:18-23.

[26] Abrams P, Chapple C, Khoury S, et al. Evaluation and Treatment of Lower Urinary Tract Symptoms in Older Men, J Urol. 2009 Apr;181(4):1779-87.

[27] Allen S, Aghajanyan IG. Benign Prostatic Hyperplasia Treatment with New Physiotherapeutic Device, Urol J, 2015 Nov 14; 12(5): 2371-2376.

[28] Allen S, Aghajanyan I. Effect of thermo balancing therapy on chronic prostatitis and chronic pelvic pain syndrome, Journal of Clinical Urology, 2016, Sept 20, 1-8, DOI: $10.1177 / 2051415816671036$.

[29] Allen S. Efficacy and safety of Thermo balancing therapy in men with lower urinary tract symptoms/benign prostatic hyperplasia was confirmed by clinical trial, 2016, ARC J of Urol, 1-1, 8-13.

[30] Allen S. The Origin of Chronic Diseases Can Be in Capillary Pathology: An Evidence from Clinical Trials on Thermobalancing Treatment of Prostate Reveals, Achievements in the Life Sciences, 2016, DOI information: 10.1016/j.als. 2016.11.005

[31] Allen S, Aghajanyan IG Thermobalancing conservative treatment for moderate-to-lowdegree lower urinary tract symptoms (LUTS) secondary to prostate enlargement, Cogent Medicine, 2016, 3(1), 1195067.

[32] Allen S, Aghajanyan IG New independent thermobalancing treatment with therapeutic device for internal diseases: chronic prostatitis and chronic pelvic pain syndrome, Nephrourol Mon,2017,e44694.doi:"http://dx.doi.org/10.581 2/numonthly.44694"10.5812/numonthly.44694.
[33] Curhan GC, Willett WC, Knight EL, Stampfer MJ Dietary Factors and the Risk of Incident Kidney Stones in Younger WomenNurses' Health Study II. Arch Intern Med, 2004, 164(8):885-891. doi:10.1001/archinte.164.8.885.

[34] Taylor EN, Stampfer MJ, Curhan GC Dietary factors and the risk of incident kidney stones in men: new insights after 14 years of follow-up. J Am SocNephrol, 2004, 15(12):3225-32. doi:10.1097/01. ASN.0000146012.44570.20.

[35] Turney BW, Appleby PN, Reynard JM, Noble JG, Key TJ, Allen NE Diet and risk of kidney stones in the Oxford cohort of the European Prospective Investigation into Cancer and Nutrition (EPIC), Eur J Epidemiol, 2014, 29, 363-369. doi:10.1007/s10654-014-9904-5.

[36] Ferraro PM, Curhan GC, Gambaro G, Taylor EN Total, Dietary, and Supplemental Vitamin C Intake and Risk of Incident Kidney Stones, Am J Kidney Dis, 2016, 67(3):400-7. doi: 10.1053/j.ajkd.2015.09.005.

[37] Coe FL, Evan A, Worcester E. Pathophysiology- based treatment of idiopathic calcium kidney stones. Clin J Am SocNephrol, 2011, 6(8):2083-92. doi: 10.2215/CJN.113212 10.

[38] Skolarikos A, Straub M, Knoll T, Sarica K, Seitz C, Petřík A, Türk C. Metabolic evaluation and recurrence prevention for urinary stone patients: EAU guidelines, EurUrol, 2015, 67(4):750-63. doi:10.1016/j.eururo.2014.10.029

[39] Gambaro G, Croppi E, Coe F et al Metabolic diagnosis and medical prevention of calcium nephrolithiasis and its systemic manifestations: a consensus statement. J Nephrol, 2016, 29(6):715-734

[40] Jung H, Andonian S, Assimos D et al Urolithiasis: evaluation, dietary factors, and medical management: an update of the 2014 SIU-ICUD international consultation on stone disease, World J Urol, 2017, 1-10. doi:10.1007 /s00345-017-2000-1.

[41] Fourcade RO, Lacoin F, Rouprêt M, et al. Outcomes and general health-related quality of life among patients medically treated in general daily practice for lower urinary tract symptoms due to benign prostatic hyperplasia. World $\mathbf{J}$ Urol. 2012; 30:419-26.

[42] Traish A, Haider KS, Doros G, Haider A. Long-term dutasteride therapy in men with benign prostatic hyperplasia alters glucose and lipid profiles and increases severity of erectile dysfunction, HormMolBiolClinInvestig. 2017, Jun21;30(3). oi: https://doi.org/10.1515/hmbci2017-0015

[43] Naeem B, Giorgio G, Akshay S et al Morbidity and Mortality After Benign Prostatic Hyperplasia Surgery: Data from the American 
College of Surgeons National Surgical Quality Improvement Program, Journal of Endourology, 2014, 28(7): 831-840.

[44] Elshal AM, Elmansy HM, Elkoushy MA, Elhilali MM. Male sexual function outcome after three laser prostate surgical techniques: a single center perspective, Urology, 2012, 80:1098-104.

[45] Brown CT, Emberton M, Self-management for men with lower urinary tract symptoms Curr prostate rep, 2009, 7: 111-116. https://doi.org/10.1007/s11918-009-0016-8.

[46] Aghajanyan, I.G.; Allen, S. Positive Response to Thermobalancing Therapy Enabled by Therapeutic Device in Men with NonMalignant Prostate Diseases: BPH and Chronic Prostatitis. Diseases 2016, 4, 18, doi:10.3390/ diseases4020018.

[47] Allen S, Aghajanyan IG Efficacy of Thermobalancing therapy for chronic prostatitis /chronic pelvic pain syndrome, confirmed by clinical study, may suggest ethology and pathophysiology of this disease, Can UrolAssoc J, 2017 Nov 11. doi: 10.5489/cuaj.4473.

[48] Allen, S. The Vascular Factor Plays the Main Role in the Cause of Pain in Men with Chronic Prostatitis and Chronic Pelvic Pain Syndrome: The Results of Clinical Trial on Thermo balancing Therapy. Diseases 2017, 5(4), 25; doi:10.3390/ diseases 5040025

[49] Allen, S.; Adjani, A. Therapeutic Device and Method, United States Patent and Trademark Office. U.S. Patent 9,408,744 B2, 9 August 2016. Available online: https://www.google. com/patents/US9408744 (accessed on 20 November 2017).

[50] Allen S, Aghajanyan IG Use of thermobalancing therapy in ageing male with benign prostatic hyperplasia with a focus on etiology and pathophysiology, Aging Male, 2016, 1-5. http://dx.doi.org/10.1080/136855 38. 2016.1247151.

Citation: Simon Allen, Thermobalancing Therapy ${ }^{\circledR}$ should be the First-Line Treatment for Kidney Stones and Benign Prostatic Hyperplasia, Discussions at the Medical Conferences. ARC Journal of Urology.2017; 2(3):1-8 doi: dx.doi.org/10.20431/2456-060X.0203001

Copyright: (c) 2017 Authors. This is an open-access article distributed under the terms of the Creative Commons Attribution License, which permits unrestricted use, distribution, and reproduction in any medium, provided the original author and source are credited. 